\title{
Selfiedontics: The Art Of Selfies Combining Cosmetic Dentistry
}

\author{
Tavane $\mathbf{P}^{1}$, Gundappa $\mathbf{M}^{2}$, Dibyendu $\mathbf{M}^{3}$, Agrawal $\mathbf{A}^{4}$, Gupta $\mathbf{S}^{5}$, Dimri $\mathbf{S}^{6}$
}

\begin{abstract}
Cosmetic dentistry has gone through potential transformations over the years. Various techniques have now been established to analyze the smile digitally and, to simulate the "Before and After" in a particular case. Selfiedontics defines the amalgamation of selfie-culture with clinical practice of dentistry. Use of selfie should not only be restricted to social platform, but also to educate the patient about his own dental status, and even in treatment planning. This article focuses on the combination of digital dentistry with that of the cosmetic or esthetic dentistry.
\end{abstract}

KEYWORDS: Cosmetic dentistry, Ideal smile, Selfiedontics, Selfie-dentistry

\author{
${ }^{1}$ Professor \\ Dept. of Conservative Dentistry and Endodontics, \\ Teerthanker Mahaveer Dental College and \\ Research Centre, Moradabad \\ ${ }^{2}$ Professor and Head \\ Dept. of Conservative Dentistry and Endodontics, \\ Teerthanker Mahaveer Dental College and \\ Research Centre, Moradabad \\ ${ }^{3}$ Professor \\ Dept. of Conservative Dentistry and Endodontics, \\ Teerthanker Mahaveer Dental College and \\ Research Centre, Moradabad \\ ${ }^{4}$ Reader \\ Dept. of Conservative Dentistry and Endodontics, \\ Teerthanker Mahaveer Dental College and \\ Research Centre, Moradabad \\ ${ }^{5}$ PG Student \\ Dept. of Public Health Dentistry, Sri Aurobindo \\ College of Dentistry, Indore \\ ${ }^{6}$ PG Student \\ Dept. of Conservative Dentistry and Endodontics, \\ Teerthanker Mahaveer Dental College and \\ Research Centre, Moradabad
}

\section{INTRODUCTION}

"Beauty is power; a smile is its sword." John Ray

B eing beautiful is powerful indeed, but combined with a smile, it is a potent combination in itself. Smile not only adds on to the esthetics but is also a profound way of communication, without words of course. Smiling has a positive influence. Researchers believe that it even has an impact over our physical and mental well being.

Many people are insecure and not confident about the way they smile. Hence, comes in the role of Cosmetic Dentistry in not only improving esthetics of a person, but also helps in boosting ones confidence level.

The role of cosmetic dentistry is to design and help enhance the facial features. As smile is one of the biggest assets constituting the esthetics, thus more symmetrical the facial features are, the higher the aesthetic appeal, and more appealing is the smile of a person.

\section{THE DIGITAL ERA}

The social media has become a tool to advertise the expertise. People are uploading near about 93 million selfies in a day. Selfies obsession is creating dental dysmorphy worldwide. Out of such a huge number, around 38\% people do not upload the pictures because they are not confident about their smile. Therefore, selfies can be used in dentistry of facial analysis and correction of smile.

\section{"Selfiedentistry / Selfiedontics is combination of cosmetic and digi- tal dentistry that is involved in the study of a selfie, diagnosis, and treat- ment planning of smile type, and also the pink esthetics, and related structures and tissues, particularly in the maxillofacial area."}

The android platform offers many apps to edit and beautify the selfies taken by people. Few of the apps such as, FaceTune, PERFECT365, \& MAKEUP PLUS gives the option to quickly retouch ones' self-portraits, whitening of teeth, refining jaw line, etc. 


\begin{tabular}{|l|l|}
\hline $\begin{array}{l}\text { The components of smile analysis and esthetic design are:-(1-6) } \\
\text { Level of aesthetics } \\
\text { 1. Facial esthetics }\end{array}$ & $\begin{array}{l}\text { Total facial form and balance. } \\
\text { 2. Oral-facial esthetics }\end{array}$ \\
\hline 3. Oral esthetics & $\begin{array}{l}\text { Maxilla-mandibular relationship to the face and the dental midline to the face pertaining to } \\
\text { the teeth, mouth, gum. }\end{array}$ \\
\hline 4. Dentogingival esthetics & Labio, dento, gingival; the relationships of the lips to the arches, gingiva and teeth. \\
\hline 5. Dental esthetics & The relationship of the gingiva to the teeth collectively and individually. \\
\hline
\end{tabular}

\section{SMILE ENGINEERING}

"A smile makeover is the process of improving the appearance of the smile through one or more cosmetic dentistry procedures, such as dental veneers, composite bonding, tooth implants and teeth whitening."

We live in the digital era, where everything can be digitalized; even the smile. The concept of smile engineering has now been introduced with smile designing techniques.

\section{AN IDEAL SMILE}

"Imperfection is beauty, madness is genius and it's better to be absolutely ridiculous than absolutely boring." -Marilyn Monroe

\section{An ideal smile constitutes of}

- The upper anterior teeth coincide with the curvature of the mandibular lip during a relaxed smile.

- The corners of the lips are elevated to the same height on both sides (termed as Smile Symmetry).

Smile can be broadly classified in to many patterns. One such classification based on the Neuromuscular pattern, which is commonly used by the plastic surgeons is:-

\section{THE COMMISSURE SMILE}

It is the most common pattern, which is seen in approximately $67 \%$ of the population. In this, the corners of the mouth are first pulled up and outward, followed by the levators of the upper lip contracting to show the upper teeth.

\section{THE CUSPID SMILE}

This smile is found in $31 \%$ of the total population. The shape of the lips are appears to be diamond shaped. This smile pattern is identified by the dominance of the levator labii superioris. They contract first, exposing the cuspid teeth, then the corners of the mouth contract to pull the lips upward and outward.

\section{THE COMPLEX SMILE}

This smile is only found in $2 \%$ of the population. The shape of the lips is typically visualized as two parallel chevrons. The levators of the upper lip, the levators of the corners of the mouth, and the depressors of the lower lip contract simultaneously, showing all the upper and lower teeth concurrently. The main feature of this smile pattern is the strong muscular pull and the retraction of the lower lip downward and backward.

Anatomically also, smile can be classified depending on the gingival line location and the anterior teeth display,

- HIGH : In this type, there is display of $100 \%$ of anterior tooth.

- MEDIUM : $75 \%$ of the tooth is visible.

- LOW : $50 \%$ or less of incisor is visible.

An esthetic smile is a balance between the facial composition and dental composition. Facial composition consists of the hard and soft tissues of the face. Dental composition constitutes more specifically of the teeth and their respective relationship to gingival tissues. The dentofacial composition encompasses both the frontal and sagittal planes in two muscular positions:-

\section{Static}

It is when the lips are slightly parted and the teeth are out of occlusion with the perioral muscles relatively relaxed.

In tranquil position, four factors mainly affect the TOOTH EXPOSURE:-
- Lip length,
- Age, COMMONLY
- Race and, KNOWN AS,
- Sex LARS'

\section{Lip Length}

The length of the upper lip varies from $10-36 \mathrm{~mm}$ and individuals with long maxillary lips show more mandibular rather than maxillary teeth. The amount of tooth exposure at rest is predominantly a muscle-determined position.

\section{Age}

Age influences the amount of tooth visibility. The amount of maxillary tooth displayed is inversely proportional to increasing age whereas the amount of mandibular teeth is directly proportional to increasing age. Therefore, a young person will display more maxillary than mandibular teeth, whereas an older individual will show more mandibular, rather than maxillary teeth.

\section{Race}

A decreasing amount of maxillary, and an increasing amount of mandibular tooth visibility, is seen from Caucasians to Asians to Blacks.

\section{Sex}

Gender differences, account for females displaying nearly twice the amount of maxillary teeth compared with men. 


\section{DYNAMIC}

The second constituent of dentofacial composition is the dynamic position characterized by a smile. The extent of tooth exposure during a smile depends on skeletal make-up, degree of contraction of the facial muscles, shape and size of the dental elements and shape and size of the lips, which vary from extremely thin to full and thick.

\section{FACIAL COMPOSITION}

The two important Facial Features in smile design: (5)

- The Inter-Pupillary Line : It should be perpendicular to the midline of the face and parallel to the occlusal plane.

- Lips : These are important since they create the boundaries of the smile design.

Lombardi suggested that a person should be viewed from the front, with facial expressions and smiling. The frontal profile of a person is the most viewed; hence, smile should be esthetically checked from this profile.

Smiling has to be considered in both horizontal as well as vertical directions. The four aspects of Smile Assessment are:-

- Vertical Dimension : Incisor display with lips at rest

- Sagittal Dimension : Assessment of incisors overjet and angulation

- Oblique Dimension : It includes the analysis of the smile arch and palatal plane direction.

- Time Factor : It includes the aspects such as growth, maturing and ageing.

The horizontal and vertical dimensions for an ideal face are as follows:

\section{Horizontal}

- The width of the face should be the width of the five eyes.

- The distance between the eyebrow and chin should be equal to the width of the face.

\begin{tabular}{|c|c|}
\hline TOOTH COMPONENTS & SOFT TISSUE COMPONENTS \\
\hline 1. Dental midline & 1. Gingival health \\
\hline 2. Incisal lengths & 2. Gingival levels and harmony \\
\hline 3. Tooth dimensions & 3. Interdental embrasure \\
\hline 4. Zenith points & 4. Smile line \\
\hline 5. Axial inclinations & \\
\hline 6. Inter-dental contact area and point & \\
\hline 7. Incisal embrasure & \\
\hline 8. Sex, personality and age & \\
\hline 9. Symmetry and balance & \\
\hline
\end{tabular}

\section{Vertical}

- The facial height is divided in to three equal parts from the forehead to the eyebrow line, from the eyebrow line to the base of the nose and from the base of the nose to the base of the chin.

- The full face is divided in to 2 parts, eyes being the midline.

- The lower part of the face from the base of the nose to the chin is divided in to 2 parts, the upper lip forms one-third of it and the lower lip and the chin forms the two-thirds.

\section{DENTAL COMPOSITION}

If considering the Dental Composition, the vital elements of smile design are:-

\section{Tooth Proportions}

The tooth proportions that are used:

- Golden proportions (Lombardi): The use of golden proportion in dental esthetics was first documented by Levin in 1978. This ratio is approximately 1:61803:1, that is, the smaller section is about $62 \%$ the size of the larger.
- Recurring esthetic dental proportions (RED/Wards): The successive width proportion when viewed from the facial aspect should remain constant as we move posteriorly from midline which offers great flexibility to match tooth properties with facial proportions.WARD.

- The values of the RED proportions used usually are between $60 \%$ and $80 \%$.

- M-proportions (Methot) : This compares the tooth width with that of the facial width using software. It involves more of mathematical calculations on a computer.(5)

- Chu's aesthetic gauges : Chu's esthetics gauges are also called as the Proportion Gauges. It enables an objective mathematical appraisal of tooth size ranges in a visual format for the clinician. (5)

\section{DIGITAL SMILE SYSTEM}

Digital Smile System is a software that allows dentists and technicians to realize the digital project of the esthetical and functional smile rehabilitation through simple and automatic tools.

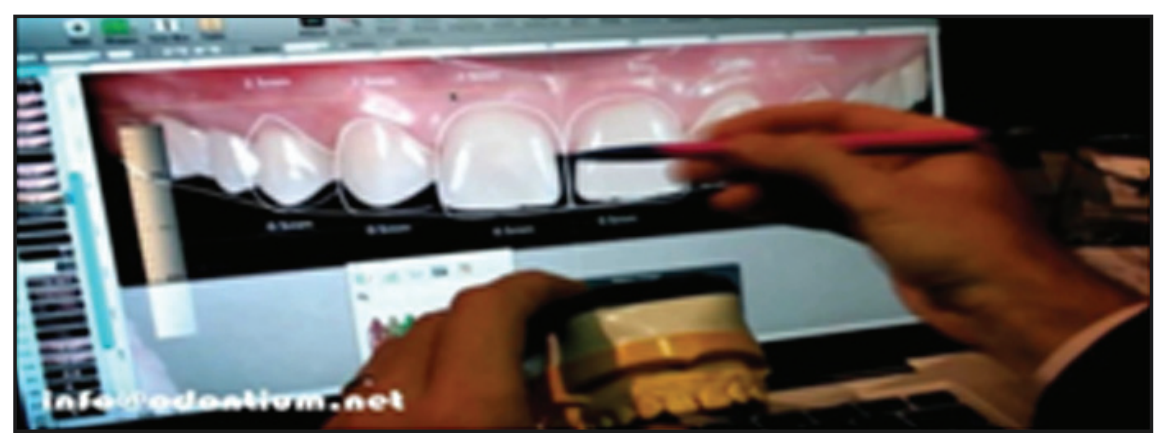


Following an intuitive and guided process dentist will be able to show to the patient the preview of the prosthetic result, giving technicians all the necessary information to realize the project.

\section{PLANMECA ROMEXIS ${ }^{\circledR}$}

It uses the Digital smile design software to manipulate the position, shape or dimensions of each tooth individually to achieve the best possible look and fit. It allows the dentists to do more than clear visualization. Completed smile designs can be fluidly exported on to any CAD/CAM software for realization.

\section{SNAP-COSMETIC SIMULATION SOFTWARE}

SNAP produces realistic cosmetic "Before and After" simulated pictures in less than a minute. This simulating software easily simulates whitening, veneers, replacing missing teeth with bridges or implants, perio-surgery and much more.

\section{GPS (GUIDE AND POSITION YOUR SMILE) 3D SMILE DE- SIGN}

GPS smile design incorporates the latest technology using digital imaging design software and the M Ruler (a patented Digital Smile Design diagnostic device).

\section{SMYLIST-PROFESSIONAL SMILE DESIGN SOFTWARE}

The aim of this software is, after reviewing the principles of custom smile design and simulating the effect of smiles with different character to decide which smile type can provide the most harmonious balance to the given face.

\section{VisagiSMile}

The software is based on innovative

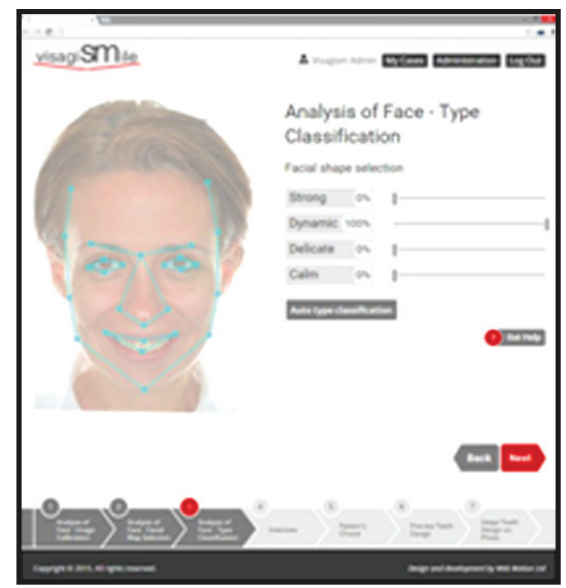

scientific theory called Dental Visagism, which explores the relationships between facial features, temperament and harmony tooth configuration of the patient.

\section{ORMCO: INSIGNIA ${ }^{\mathrm{TM}}$ AD- VANCED SMILE DESIGN ${ }^{\text {TM }}$ SOFTWARE}

This software allows combining treatment strategy with the precision of computer-aided smile design to provide each patient an optimal occlusion and smile arc specifically proportioned to their unique facial features.

\section{CEREC SW 4.2 SMILE DESIGN}

The CEREC 4.2 software can design smiles by simply taking a full face smile photograph and using it with the new Guideline and Incisal Variation features.

\section{OTHERS}

Dental Magic by Dental Master, NemoDSD

\section{SMILE DESIGNING TECH- NIQUES}

After careful analysis of the case, various techniques can be then used to design the smile

- Tooth whitening/bleaching

- Crowns
- Enamel shaping

- Gum contouring

- Veneers

- Lumineers

\section{CONCLUSION}

On the basis of above discussion, we can conclude that careful evaluation and analysis of the smile pattern is needed to successfully design a smile. The dental profession has experienced an appreciable amount of technological growth. With the incorporation of digital or computer-controlled components, the clinicians are now able to achieve better vision and enhanced precision.

Brands like Denplan, U.K introduced the concept of \#doityourselfies. This ad connects the importance of good oral health and visiting a dentist with the current social phenomenon of the "Selfie Culture". Hence, the concept of selfies should be applied not only to educate the patient and make him aware of his dental status, but also, as a means of analyzing, evaluating and predicting the results.

\section{REFERENCES}

1. Douglas A. Terry and Willi Geller: Esthetic and Restorative Dentistry-2 ${ }^{\text {nd }}$ edition (Quintessence Publishing)

2. George A. Freedman: Contemporary Esthetic Dentistry.

3. Adam J Mieleszko, Alessandro Devigus, Stephen J Chu. Fundamentals of Colour, Shade Matching and Communication in Esthetic Dentistry $2^{\text {nd }}$ edition

4. Marc Geissberger: Esthetic dentistry in Clinical Practice

5. Mohan Bhuvaneswaran: Principles of smile design. J Conserv Dent 2010;13(4): 225-32.

6. Smile analysis. The Photoshop $\AA$ smile design technique:Part I-Edward A. McLaren, DDS; Lee Culp, CDT, AAACD, Spring 2013, Volume 29, Number 1. 